\title{
Identification of the Products of Oxidation of Quercetin by Air Oxygen at Ambient Temperature
}

Igor G. Zenkevich ${ }^{1, *}$, Anna Yu. Eshchenko ${ }^{2}$, Svetlana V. Makarova ${ }^{2}$, Alexander G.

Vitenberg ${ }^{1}$, Yuri G. Dobryakov ${ }^{1}$ and Viktor A. Utsal ${ }^{3}$

${ }^{1}$ St. Petersburg State University, Research Institute of Chemistry, Universitetsky pr., 26, St.

Petersburg 198504, Russia; E-mails: vitenberg@mail.ru; yura@nonel.pu.ru

2 Interregional Center “Adaptogen”, Piskarevsky pr., 47/5, St. Petersburg 195067, Russia; E-mails: anna_eshenko@mail.ru; utsedus@yandex.ru

3 Russian Scientific Center “Applied Chemistry”, St. Petersburg 197198, Dobrolubov ave., 14, Russia; E-mail: utsal@bk.ru

* Author to whom correspondence should be addressed; E-mails: igor@IZ6246.spb.edu; izenkevich@mail15.com

Received: 13 January 2007; in revised form: 22 March 2007 / Accepted: 25 March 2007 / Published: 27 March 2007

\begin{abstract}
Oxidation of quercetin by air oxygen takes place in water and aqueous ethanol solutions under mild conditions, namely in moderately-basic media $(\mathrm{pH} \sim 8-10)$ at ambient temperature and in the absence of any radical initiators, without enzymatic catalysis or irradiation of the reaction media by light. The principal reaction products are typical of other oxidative degradation processes of quercetin, namely 3,4-dihydroxy-benzoic (protocatechuic) and 2,4,6-trihydroxybenzoic (phloroglucinic) acids, as well as the decarboxylation product of the latter - 1,3,5-trihydroxybenzene (phloroglucinol). In accordance with the literature data, this process involves the cleavage of the $\gamma$-pyrone fragment (ring $C$ ) of the quercetin molecule by oxygen, with primary formation of 4,6dihydroxy-2-(3,4-dihydroxybenzoyloxy)benzoic acid (depside). However under such mild conditions the accepted mechanism of this reaction (oxidative decarbonylation with formation of carbon monoxide, $\mathrm{CO}$ ) should be reconsidered as preferably an oxidative decarboxylation with formation of carbon dioxide, $\mathrm{CO}_{2}$. Direct head-space analysis of the gaseous components formed during quercetin oxidation in aqueous solution at ambient temperature indicates that the ratio of carbon dioxide/carbon monoxide in the gas phase after acidification of the reaction media is $c a .96: 4 \%$. Oxidation under these mild conditi-
\end{abstract}


ons is typical for other flavonols having $\mathrm{OH}$ groups at $\mathrm{C}^{3}$ (e.g., kaempferol), but it is completely suppressed if this hydroxyl group is substituted by a glycoside fragment (as in rutin), or a methyl substituent. An alternative oxidation mechanism involving the direct cleavage of the $C^{2}-C^{3}$ bond in the diketo-tautomer of quercetin is proposed.

Keywords: Quercetin; oxidative decarboxylation; air oxygen; mechanism; products; identification

\section{Introduction}

Quercetin [3,5,7,3',4'-pentahydroxyflavone, 5,7,3',4'-tetrahydroxyflavonol (1)] and its glycosides [e.g., 3-O-rhamnoside (quercitrin) and especially 3-O-rutinoside (rutin)] are among the most frequently mentioned natural flavonoids isolated from various plant extracts [1-3].<smiles>O=c1c(O)c(-c2ccc(O)c(O)c2)oc2cc(O)cc(O)c12</smiles>

1

This statement can be easily illustrated by the frequency of the citation of the names of different flavonoids on the Internet (Google search, November 2006), summarized in Table 1.

Table 1.

\begin{tabular}{l|c|c}
\hline Flavonoid & $\begin{array}{c}\text { Number of } \\
\text { search “hits” }\end{array}$ & $\begin{array}{c}\text { Ratio to quercetin, } \\
\text { \% }\end{array}$ \\
\hline Kaempferol & 214,000 & 14 \\
Luteolin & 17,400 & 1 \\
Fisetin & 59,600 & 4 \\
Daidzein & 349,000 & 22 \\
Myricetin & 97,600 & 6 \\
Quercetin & $1,580,000$ & 100 \\
Rutin & $3,060,000$ & 194 \\
\hline
\end{tabular}

Current interest in the compounds of flavonoid group, including 1, is explained by their varied biological activity, with antioxidant, antiradical, hepatoprotective, anti-inflammatory and other interesting properties [4-8].

If $\mathbf{1}$ is such a widespread compound, its properties, physicochemical constants and analytical parameters should be described in detail. Its "traditional" characteristics, used in the identification of this compound, are its melting point $\left(313-315^{\circ} \mathrm{C}\right)$ and the variations of its UV-spectra at the presence 
of different reagents [1,2]. The range of additional analytical parameters available includes relative UV-absorption abundances at various wavelengths [9,10], retention indices (RI) in reversed-phase HPLC [11] and gas chromatographic RIs of the trimethylsilyl (TMS) derivatives of 1 on standard nonpolar polydimethyl siloxane phases (original data, Tab1e 2).

Table 2.

\begin{tabular}{l|c}
\hline \multicolumn{1}{c|}{ TMS-derivative of $\mathbf{1}$} & RI \\
\hline 5,7,3', $4^{\prime}$-tetra-TMS & $3011 \pm 3$ \\
3,7,3',4' -tetra-TMS & $3092 \pm 3$ \\
penta-TMS & $3171 \pm 4$ \\
\hline
\end{tabular}

Only as recently as 2003 was the anomalous UV-spectrum of the pentaacetyl derivative of $\mathbf{1}$ reported and explained; this spectrum is very different from those of the quercetin parent compound and all other acetyl derivatives (from mono- to tetra-) [12].

One of principal chemical property of flavonols [including quercetin (1)] seems to be their facile oxidation by air oxygen, which has been the subject of numerous publications to date. In particular the first report on the oxidation of quercetin was that by Nierenstein in 1912 [13]. This interest can be verified by the number of corresponding search "hits" on the Internet. However, in most of these reports the oxidation of quercetin was preferably carried out in strongly basic organic solvents or their mixtures with water under the influence of various additional factors, namely heating of reaction media, the presence of radical initiators, under enzymatic catalysis and light or $\gamma$-irradiation. The possible mechanism of photosensitized oxygenation of 3-hydroxyflavones by air [bubbled through their pyridine solutions under irradiation by light] was proposed first by Matsuura et al. [14,15]. It includes formation of peroxide intermediates of the 3-hydroxyflavones, followed by their conversion into the corresponding depsides and the subsequent hydrolysis products (see discussion below), resulting in the cleavage of ring $\mathrm{C}$. Both carbon monoxide $(\mathrm{CO})$ and carbon dioxide $\left(\mathrm{CO}_{2}\right)$ were detected in different proportions among the gaseous products of this reaction, depending on the conditions used. These results suggest that the reaction may actually proceed simultaneously through two different mechanisms. Only one of these possible mechanisms (involving formation of carbon monoxide) was accepted later; it was confirmed with the use of isotope labeled oxygen $\left({ }^{18} \mathrm{O}_{2}\right)[16]$.

Similar processes of oxidative destruction of 3-hydroxyflavones (including quercetin) by air oxygen with the formation of the same products were observed under catalysis by the enzymes quercetinase [16] and tyrosinase [17], in the presence of radical initiators like azodiisobutyronitrile [18-20] or the $\mathrm{CuSO}_{4} / \mathrm{H}_{2} \mathrm{O}_{2}$ system [21], and in the complexes of flavonols with $\mathrm{Cu}^{2+}$ ions [22,23]. The same degradation products were detected as a result of $\gamma$-irradiation of quercetin solutions [24,25] and even heating of its solutions at the presence of oxygen [26,27]. Under some conditions the formation of other products was observed. Namely, electrochemical two-electron oxidation of (1) does not give depside, but rather 2,4,6-trihydroxy-2-(3,4-dihydroxybenzoyl)-3(2H)-benzofuranone [28]. The same benzofuranone, together with depside, was detected among the products of reaction of $\mathbf{1}$ with azodiisobutyronitrile [19].

The comparison of the various processes of quercetin oxidation by air allows us to conclude that the features of this reaction under the mildest conditions, i.e. in water and water-ethanol solutions in moderately basic media ( $\mathrm{pH} \sim 8-10$ ) at ambient temperature (i.e. without heating) in the absence of 
radical initiators, any complexing reagents or fermentative catalysis and without $\gamma$ - or light irradiation of the reaction media are still not well-characterized at present. The first experiments indicated that quercetin was quickly destroyed by simply bubbling air through its solutions under such mild conditions. This allows us to classify $\mathbf{1}$ as more unstable than the well-known ascorbic acid, which is inert to contact with air under the same conditions. Of course, there is a high probability that the products of this reaction should be the same as established previously [14-27], but this should be confirmed independently. In addition there is the problem is the origin of gaseous products formed in this reaction ( $\mathrm{CO}$ or $\mathrm{CO}_{2}$ ), because the oxidative state of carbon in these oxides determines the possible mechanism of the process.

Hence, the aims of this paper were the identification of the quercetin oxidation products formed under as mild conditions as possible, including analysis of the resulting the gas phase carbon oxides in the reaction media. Preliminary data on the instability of quercetin solutions at the contact with air under such mild conditions have been reported [29].

\section{Results and Discussion}

\section{Preliminary experiments}

Easy oxidation of quercetin (1) by air oxygen in the moderate-alkaline solutions at ambient temperature should be considered as one of most important chemical properties of flavonols. A simple experiment, namely the slow stepwise titration of $\mathbf{1}$ in $30 \%$ water ethanol solution by sodium hydroxide within $\mathrm{pH}$ range from 2 to 10 (Figure 1), confirms the universal character of this process.

Figure 1. Variations of optical densities of quercetin solution at wavelength $294 \pm 2 \mathrm{~nm}$ in result of its slow stepwise titration by $\mathrm{NaOH}$ within the $\mathrm{pH}$ range $6-10$. The anomaly at $\mathrm{pH}$ 7.2-7.1 is caused by the interruption of the process for $c a$. 30 min; the second one (at $\mathrm{pH}$ 8.2-7.7) was obtained after the storage of the solution during one week.

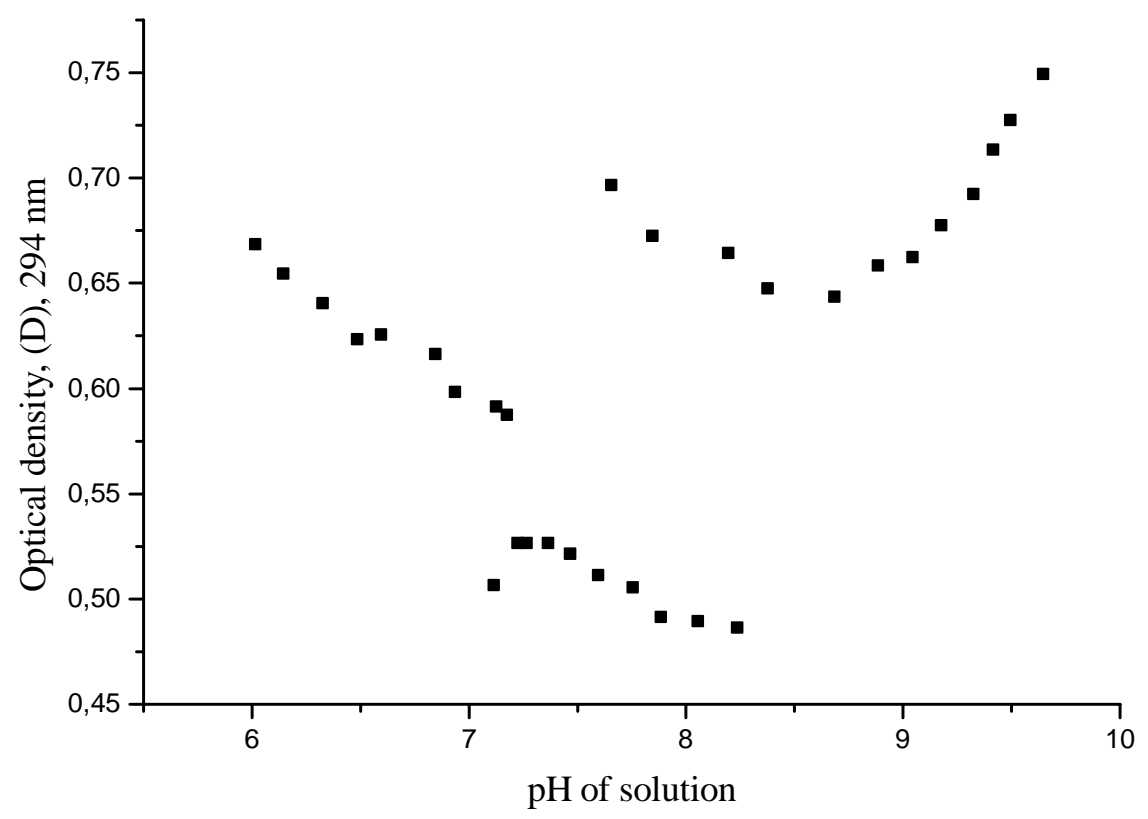


After addition of each portion of titrant, the $\mathrm{pH}$ of solution was measured and its UV-spectrum recorded. Surprisingly, any interruption of this uniform procedure leads to a gap in the smooth dependence of $\mathrm{pH}$ vs. volume of titrant curve and disruptions in the UV-abundances at different wavelengths. These anomalies in the $\mathrm{pH}-\mathrm{D}(294 \mathrm{~nm})$ coordinates are clearly evident in Figure 1.

The first anomalous area at $\mathrm{pH} \approx 7.2-7.1$ was caused by the storage of the solution under titration during $c a$. $30 \mathrm{~min}$; the second one (at $\mathrm{pH} \approx 8.2-7.7$ ) was caused by its storage for one week. The reproducibility of these effects permits us to exclude the influence of experimental errors and conclude the existence of an actual experimental feature. The complete UV-spectra of 1 during titration within the $\mathrm{pH}$ range 2-10 indicate anomalies as well. Some of them can be explained by the acid-base equilibrium $\mathrm{ArOH} \leftrightarrow \mathrm{ArO}^{-}\left[\mathrm{pK}_{\mathrm{a}}(\mathbf{1})=7.3 \pm 0.1\left(\mathrm{OH}^{7}\right)\right]$ [30]. However, the acidification of the basic solution $(\mathrm{pH}=10)$ after slow stepwise titration did not restore its initial UV-spectrum (Figure 2). At the same time, the quick reversible $\mathrm{pH}$ variation (in only two steps) in accordance with the scheme “acid $\rightarrow$ basic $\rightarrow$ acid” restores a UV-spectrum identical to that of initial solution.

Figure 2. Variations of the UV-spectra of a water-ethanol solution of quercetin during its slow stepwise titration by $\mathrm{NaOH}$ within $\mathrm{pH}$ range 2-10, followed by the acidification up to $\mathrm{pH}=4$; (a) $-\mathrm{UV}$-spectrum of quercetin in ethanol solution; (b) - at $\mathrm{pH}=2$; (c) - at $\mathrm{pH}=10$; (d) - UV-spectrum of solution after acidification to $\mathrm{pH}=4$.

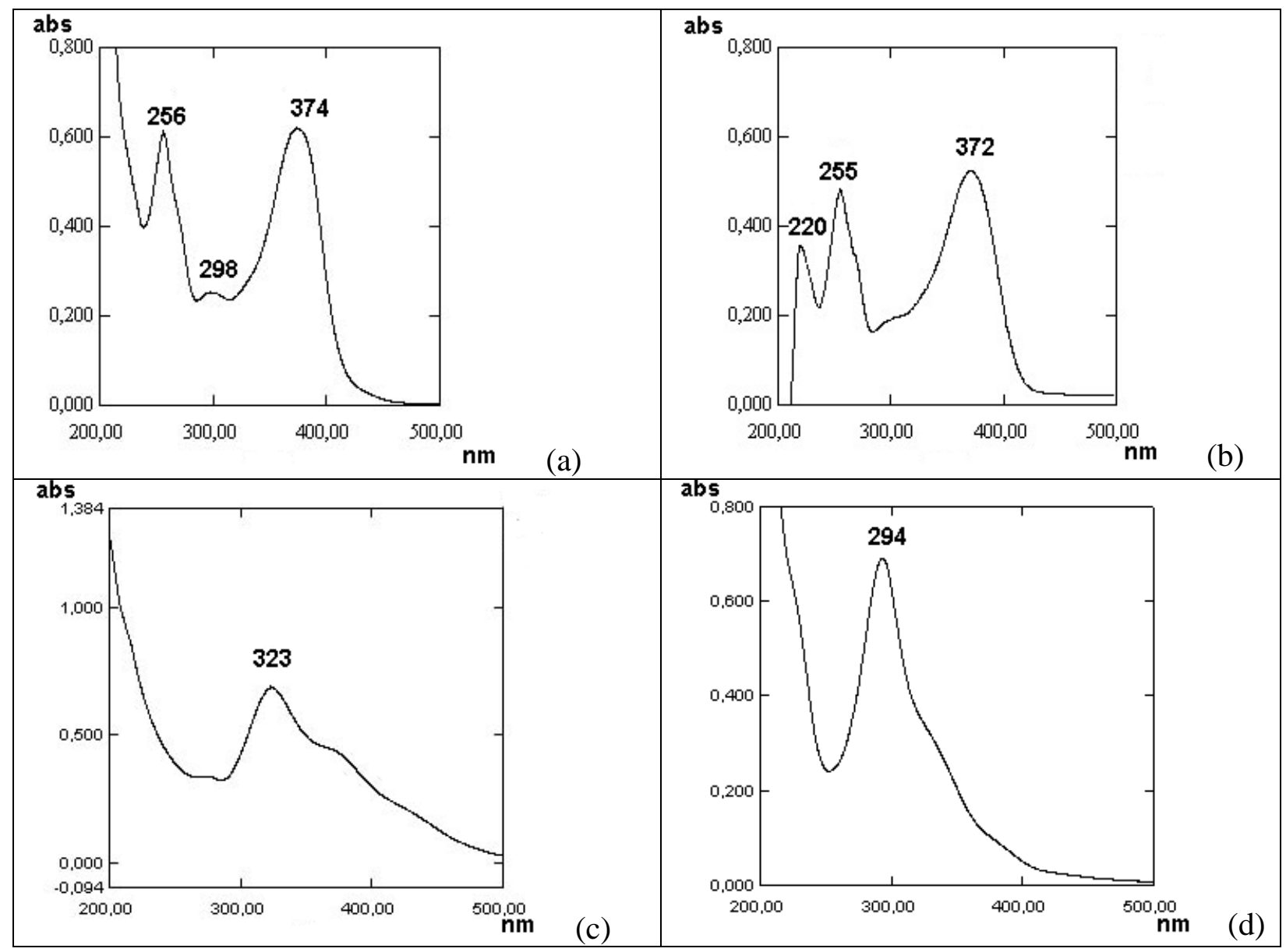


All the mentioned effects can be explained by the instability of quercetin (1) towards the oxidation by air oxygen during slow titration and all other operations involving its dilute solutions at ambient temperature. The oxygen ground state is the triplet one. This means that the $\mathrm{O}_{2}$ molecule exists as a reactive biradical ['O-O']. The solubility of oxygen in the water at ambient conditions ( 760 torr, $20^{\circ} \mathrm{C}$ ) is about $8.8-9.4 \mathrm{mg} / \mathrm{L}[31,32]$ or about $0.29 \mathrm{mmol} / \mathrm{L}$. This reference value can be accepted without additional verification and it should remain constant when air is bubbling through aqueous reaction media. For comparison, the concentration of $\mathbf{1}$ in the solution used for recording of UV-spectrum was ca. $20 \mathrm{mg} / \mathrm{L}$, or $0.06 \mathrm{mmol} / \mathrm{L}$. That is, there was at least a 4.5 -fold molar excess of oxygen in this solution.

Figure 3. HPLC chromatograms (regime 1, detection wavelength $254 \mathrm{~nm}$ ) (a) - initial solution of quercetin ( $\mathrm{t}_{\mathrm{R}} 15.2 \mathrm{~min}$ ); (b) - the same solution after $\sim 1 \mathrm{~h}$ of air bubbling at ambient temperature; (c) - the same solution after termination of the process (air bubbling during more than 3-3.5 h).

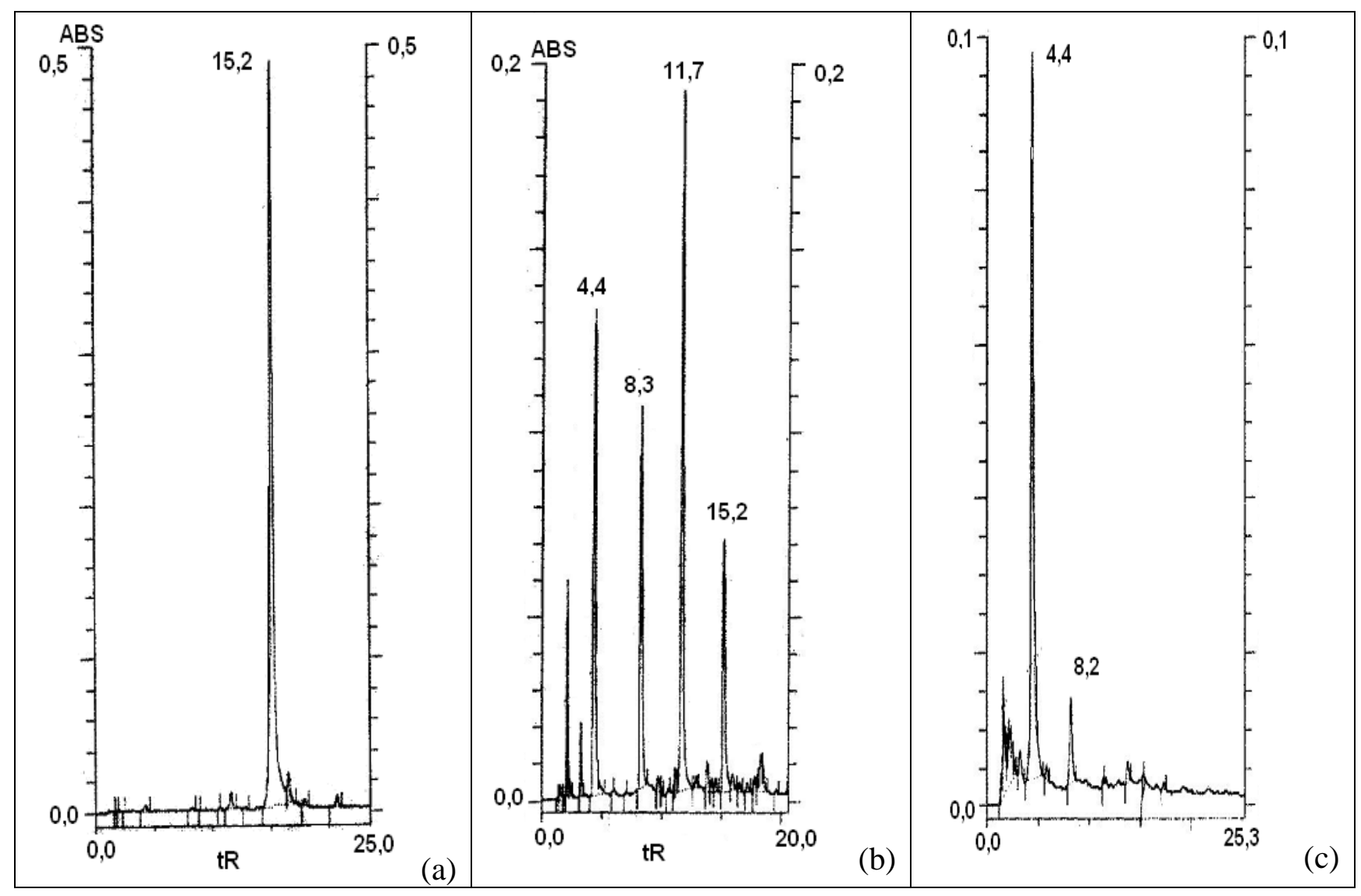

Experimental oxidation of $\mathbf{1}$ by air was carried out using a simple procedure. Air was bubbled through water-ethanol solutions $(\mathrm{pH} \approx 8-10)$ of 1 at ambient temperature during up to $3.5 \mathrm{~h}$ with periodical HPLC analyses of the reaction medium at different times. The chromatograms of the initial quercetin solution (individual compound with $t_{R} 15.2 \mathrm{~min}$, regime 1 ) after $1 \mathrm{~h}$ of air bubbling and that after termination of the process ( $3.5 \mathrm{~h}$ ) are compared in Figure 3. No peak of $\mathbf{1}$ is observed on the final chromatogram; only the signal of a hydrophilic compound(s) with $t_{R} c a .4 .4 \mathrm{~min}$ is registered. No additional changes have been detected with the increase of the contact time of this solution with air. The same transformations after a similar period are observed when the reaction medium was cooled 
down to $0{ }^{\circ} \mathrm{C}$, which is typical for radical processes. Oxidation of $\mathbf{1}$ by air oxygen is accompanied by the appearance of an intense red-brown coloration of the solution. The dry product isolated from this solution after evaporation of the solvents possesses the same color.

Analysis of oxidation products

HPLC analysis of dry mixture of oxidation products (regime 2) indicate the presence of four principal products with retention times of 4.15, 4.80, 6.05 and 20.97 min (initial quercetin with $t_{R} 25.7$ min is absent in this mixture), as shown in Figure 4. By analogy with literature data it seems reasonable to believe that oxidation products of $\mathbf{1}$ possess simpler chemical structures than that of the initial compound. So far as quercetin itself can be analyzed in the form of its trimethylsilyl (TMS) derivatives (see their RI values in Table 1), GC-MS analysis of TMS-derivatives of oxidation products can be used as the method for their identification. Three principal compounds accounting for ca. $96 \%$ among semi-volatile substances were found in the dry mixture of oxidation products (Table 3).

Figure 4. HPLC chromatogram (regime 2) of dried quercetin oxidation products (initial compound with $\mathrm{t}_{\mathrm{R}} 25.68 \mathrm{~min}$ is absent). Component with $\mathrm{t}_{\mathrm{R}} 4.15 \mathrm{~min}$ - phloroglucinol; $4.890 \mathrm{~min}$ - 2,4,6-trihydroxybenzoic acid; $6.05 \mathrm{~min}$ - 3,4dihydroxybenzoic acid; 12.02 - intermediate depside (2); $20.97 \mathrm{~min}$ unidentified compounds. Each peak is $100 \%$ pure as there is no co-elution of compounds.

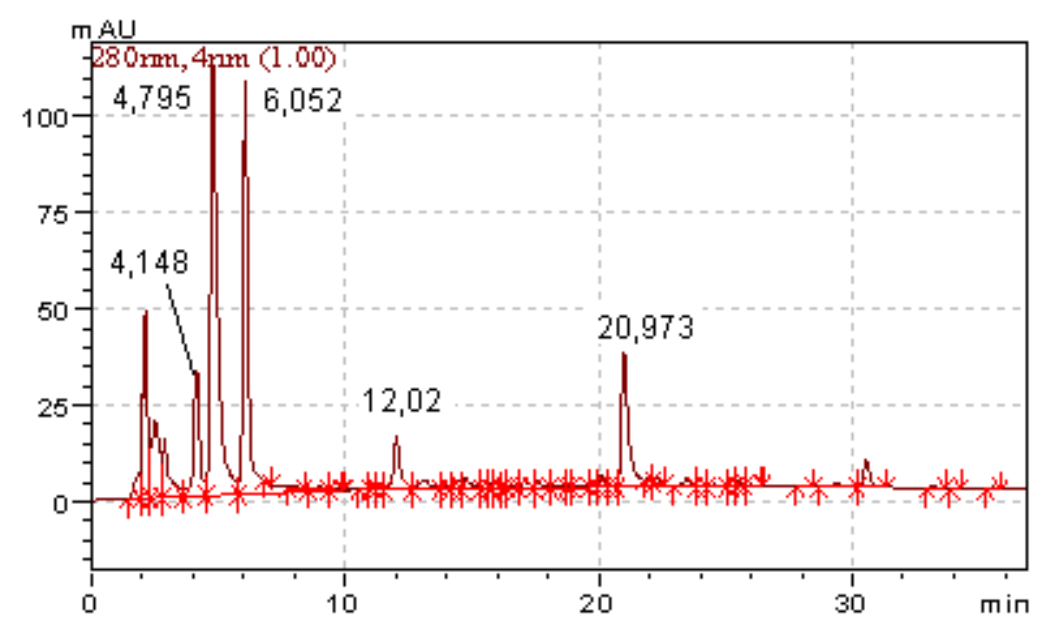

Table 3.

\begin{tabular}{c|c|l|l}
\hline $\begin{array}{c}\mathbf{t}_{\mathbf{R}}, \\
\text { min }\end{array}$ & $\begin{array}{c}\text { Relative } \\
\text { content, \% }\end{array}$ & \multicolumn{1}{|c}{ MS identification } & Reference RI values \\
\hline 25.48 & 10 & Phloroglucinol, tris-TMS (A) & 1656 \\
27.71 & 1 & Ethyl-3,4-dihydroxybenzoate, bis-TMS & - \\
28.25 & 53 & 3,4-Dihydroxybenzoic acid, tris-TMS (B) & 1830 \\
30.72 & 33 & 2,3,4-Trihydroxybenzoic acid, tetrakis-TMS (C) & 1932 (2,3,4-isomer); \\
& & & 2003 (2,4,6-isomer) \\
32.34 & 2 & Unidentified & - \\
\hline
\end{tabular}


Components $\mathbf{A}$ and $\mathbf{B}$ can be identified ambiguously with the use of the Wiley 138.L MS database: they are TMS-derivatives of 1,3,5-trihydroxybenzene (phloroglucinol, A) and 3,4-dihydroxybenzoic (protocatechuic) acid (B). The third component $\mathbf{C}$ was tentatively identified as the TMS derivative of 2,3,4-trihydroxybenzoic acid, but this is not consistent with the absence of a structural fragment with this arrangement of hydroxyl groups in the molecule of $\mathbf{1}$. A more reliable assignment of the structure of this oxidation product is 2,4,6-trihydroxybenzoic (phloroglucinic) acid, which is in agreement with the position of substituents on ring A of the initial molecule. The erroneous GC-MS identification of this compound is likely caused by the absence of its mass spectrum in the Wiley MS database; as a result it is recognized as an isomer. The value of GC retention index of this TMS-derivative (1996) is in the better agreement with reference RI value for TMS derivative of 2,4,6-trihydroxybenzoic acid (2003 \pm 9) than with that for 2,3,4-trihydroxybenzoic acid (a single reference value 1932 is available).

The comparison of GC-MS results with the data of HPLC analysis (Figure 4) permits us to identify 3,4-dihydroxybenzoic acid (B) by the coincidence of its retention time (6.05 $\mathrm{min}$ ) and UV-spectrum ( $\lambda_{\max }$ 216, 259 and $293 \mathrm{~nm}$ ) with a reference sample. More hydrophilic components with retention times 4.15 and $4.80 \mathrm{~min}$ are phloroglucinol (A) and 2,4,6-trihydroxybenzoic acid (C), respectively. The relatively hydrophobic compounds with retention times 12.02 and $20.97 \mathrm{~min}$ have no matches in the GC-MS analysis results and therefore cannot be identified this way. One of them (with $t_{R} 8.3 \mathrm{~min}$ in regime 1 and $12.02 \mathrm{~min}$ in regime 2) is the intermediate depside (ester) that is unstable to hydrolysis in a basic media.

The retention indices of compounds $\mathbf{B}$ and $\mathbf{C}$ in reversed phase HPLC (with the use of $n$-alkyl phenyl ketones as reference compounds) have been determined first time; both of them are in the accordance with the previously reported RI value of phloroglucinol [11], because the ascending order of RI values reflects the order of chromatographic elution (Table 4). The same products were isolated in the result of quercetin oxidation by hydrogen peroxide under the same $\mathrm{pH}$ conditions.

Table 4.

\begin{tabular}{l|c}
\hline \multicolumn{1}{c|}{ Compound } & RI \\
\hline Phloroglucinol (A) & $582[11]$ \\
2,4,6-Trihydroxybenzoic acid (C) & 606 \\
3,4-Dihydroxybenzoic acid (B) & 618 \\
\hline
\end{tabular}

The formation of the aforementioned products is in complete agreement with literature data on the oxidation of quercetin under different conditions [14-27]. To date the oxidative decarbonylation of 1 was considered the likely process which could take place preferably in non-aqueous solvents in the presence of strong bases and often at above-ambient temperatures (in pyridine during $14 \mathrm{~h}$ [14], in

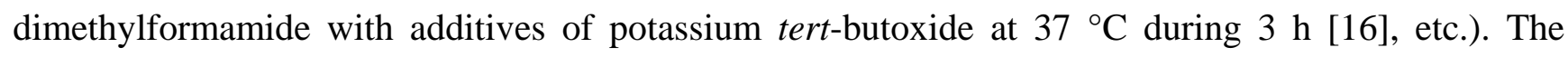
accepted mechanism of this reaction (Scheme 1) includes the attack of carbon atom $\mathrm{C}^{2}$ in ring $\mathrm{C}$ by oxygen with formation of a hydroperoxide anion. The cyclization of this anion implies the formation of a new $\mathrm{O}-\mathrm{C}^{4}$ bond, followed by elimination of the $\mathrm{C}^{3}$ carbon atom in the form of carbon monoxide (CO). The resulting primary product is 4,6-dihydroxy-2-(3,4-dihydroxybenzoyloxy)benzoic acid (depside, 2, CAS \# 30048-34-1); its subsequent hydrolysis in basic media gives salts of 3,4-dihydroxyand 2,4,6-trihydroxybenzoic acids. Compound $\mathbf{2}$ has been identified as one of the intermediate 
products of quercetin oxidation (component with $t_{R} 8.3 \mathrm{~min}$ and $12.02 \mathrm{~min}$ on the chromatograms presented in Figures 3 and 4, correspondingly).

Scheme 1. Possible mechanism of quercetin oxidative decarbonylation by oxygen [16].<smiles>O=c1c([O-])c(-c2ccc(O)c(O)c2)oc2cc(O)cc(O)c12</smiles><smiles>CC1(C)OC2(c3ccc(O)c(O)c3)C(=O)C(=O)C1(O[O-])Oc1ccc(O)cc12</smiles>

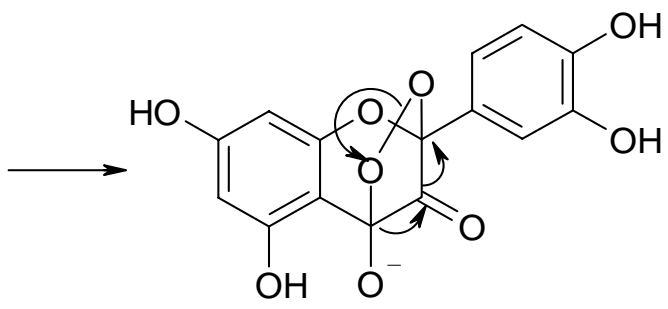<smiles>O=C(Oc1cc(O)cc(O)c1C(=O)[O-])c1ccc(O)c(O)c1</smiles>

This mechanism was partially confirmed by isotope labeling using of ${ }^{18} \mathrm{O}_{2}$ for oxidation [16]. The isotope labels were found distributed in a 1:1 ratio in both acids formed after hydrolysis of depside (2). Nevertheless, the oxidative decarbonylation of quercetin presented in Scheme 1 seems to not be the only possible mechanism. First, as reported in the publications of Matsuura et al. [14,15], carbon monoxide may not be the sole gaseous product of this reaction; the formation of carbon dioxide was detected as well. The $\mathrm{CO}_{2}$ : $\mathrm{CO}$ ratio was about 0.55 if the reaction media (pyridine solution) was irradiated by a tungsten lamp, but it exceeds 1 under UV-irradiation (when a high-pressure mercury lamp with Pyrex filter was used). In accordance with Scheme 1, the ratio of reacted oxygen and formed carbon monoxide should be 1:1, i.e., if the oxidation of $\mathbf{1}$ is carried out in a closed system, the total gas pressure should remain constant.

Quercetin (like other flavonoids) with a $\gamma$-pyrone ring in the molecule (ring C) can exist in two prototropic forms (Scheme 2); the first of them is well-known enolic structure the $\gamma$-pyrone molecular fragment, while the second one is the tautomeric diketoform 3 (the ratio of tautomers is unknown and it can depend on the $\mathrm{pH}$ of the solution)

Scheme 2. Keto-enol tautomerisation of quercetin.<smiles>O=c1c(O)c(-c2ccc(O)c(O)c2)oc2cc(O)cc(O)c12</smiles><smiles>C=C</smiles><smiles>O=C1C(=O)C(c2ccc(O)c(O)c2)Oc2cc(O)cc(O)c21</smiles> 
The existence of tautomer $\mathbf{3}$ was taken into account in the quantum chemical modeling of the oxidative properties of $\mathbf{1}$ [33]. It is interesting to note that one of the principal chemical features of $\alpha$ diketones is their easy oxidation in basic media by air oxygen (Scheme 3). This reaction goes via intermediate ketyl anion-radicals [34] and gives two carboxylic acids in result of C-C bond cleavage $[35,36]$. It was characterized in details on the example of 2,2,5,5-tetramethylfuranidin-3,4-dione [35]:

Scheme 3. Example of the oxidative cleavage of $\alpha$-diketones.<smiles>CC1(C)OC(C)(C)C(=O)C1=O</smiles><smiles>CCOC(=O)O</smiles><smiles>CC(C)(C)OC(C)(C)C(=O)[O-]</smiles>

A similar scheme for oxidation of another $\alpha$-diketo compound (dehydroascorbic acid) by superoxide anion-radicals $\left(\mathrm{O}_{2}{ }^{-}-\right.$one of the forms of active oxygen in chemical reactions) with the cleavage of $\mathrm{C}-\mathrm{C}$ bong and formation of two carboxylic acids was proposed by Frimer and GilinskySharon in 1995 [37] (Scheme 4).

Scheme 4. Oxidative cleavage of dehydroascorbic acid by $\mathrm{O}_{2}{ }^{-}$

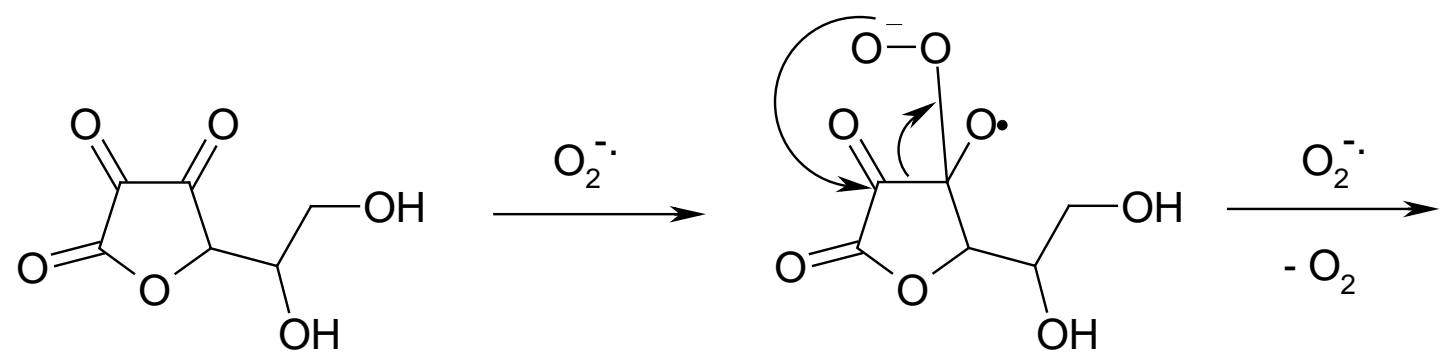<smiles>CCCCC(C)O</smiles>

Keeping in mind these properties of $\alpha$-diketones and applying them to the quercetin oxidation permits us to propose the alternative mechanism of the process with the formation of the same depside 2 shown in Scheme 5.

In comparison with Scheme 1 this is not oxidative decarbonylation, but rather what has taken place is a decarboxylation (accompanied by $\mathrm{CO}_{2}$ formation). Therefore, more than one equivalent of oxygen is required for the formation of one equivalent of carbon dioxide. As far as this process takes place in basic media, the carbon dioxide formed immediately converts into $\mathrm{CO}_{3}{ }^{2-}$ ions in the solution; hence no elimination of gases from the reaction media should be observed. The radical character of this reaction is confirmed by the absence of changes in its rate as a result of the cooling of the quercetin solution. 
Scheme 5. Alternative mechanism of quercetin oxidative decarboxylation by oxygen.<smiles>O=C1C(=O)c2c(O)cc(O)cc2OC(O[O-])(c2ccc(O)c(O)c2)C1=O</smiles>

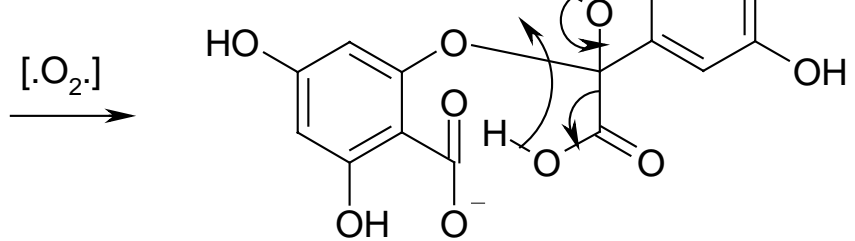

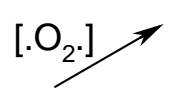

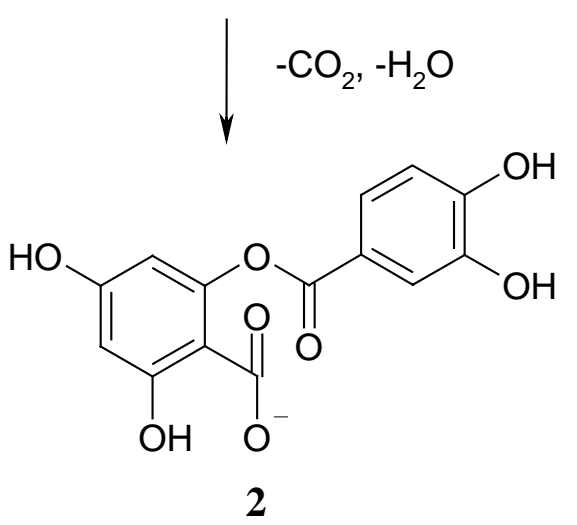

It is important to note that Scheme 5 should be in the agreement with the observed distribution of ${ }^{18} \mathrm{O}$ isotope labels among products as Scheme 1 as well. The primary product of this reaction is the same depside 2; its subsequent hydrolysis in basic media gives two hydroxybenzoic acids. Besides that, the hydrolysis of $\mathbf{2}$ in water-ethanol mixtures gives small amounts of an ethyl ester (ethyl-3,4dihydroxybenzoate); this compound was detected with GC-MS analysis of the mixture of reaction products. The presence of variable amounts of phloroglucinol (A) among the isolated quercetin oxidation products can be explained by the easy decarboxylation of 2,4,6-trihydroxybenzoic acid (B) during drying of reaction mixtures (at the temperature ca. $100^{\circ} \mathrm{C}$ ) (Scheme 6).

Scheme 6. Formation of phloroglucinol in the result of decarboxylation of 2,4,6trihydroxybenzoic acid.<smiles>O=C(O)c1c(O)cc(O)cc1O</smiles>

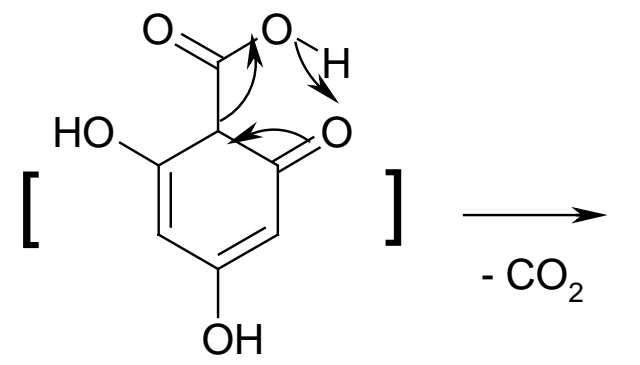<smiles>Oc1cc(O)cc(O)c1</smiles>

Unfortunately, the chemical origin of the compound(s) responsible for the appearance of intensive color of reaction media and isolated dry products remains unknown. It is reasonable to assume that these compound(s) should include benzoquinone-like molecular fragments (chromophores), but its (their) content may be small. The screening of UV-spectra of all components detected during HPLC analysis with diode array detector in relation to the absorbance in the visible region gave no results. Both Schemes 1 and 5 confirm that the principal structural fragment of the quercetin molecule that is responsible for the easy oxidation of this compound by air oxygen is the hydroxyl group at $\mathrm{C}^{3}$. The 
substitution of this group by glycoside fragments completely suppresses the oxidation. For example, rutin becomes stable, while another flavonol (3,5,7,4'-tetrahydroxyflavon, kaempferol) oxidizes easily under the same conditions. This fact is in accordance with the literature data, whereby 3-O-methylated flavonols gave no oxidation products in the presence of radical initiators [18].

Therefore, the selection of the reliable mechanism of quercetin oxidation by air oxygen seems impossible without direct analysis of gaseous products $\left(\mathrm{CO}\right.$ or $\left.\mathrm{CO}_{2}\right)$ formed in this process in the gas phase contacting with reaction media (so-called head-space analysis, HSA [37]). This experiment was carried out in closed system containing $1 \mathrm{~L}$ of water quercetin solution $(2.1 \mathrm{~g}, 6.2 \mathrm{mmol} / \mathrm{L}, \mathrm{pH} \sim 11$, basified by $\mathrm{NaOH}$ ) and $2 \mathrm{~L}$ of air and a digital manometer for pressure measurements. The analysis of gas phase was conducted with the use of gas chromatograph equipped by two packed columns placed in parallel and thermo conductivity detector (carrier gas was hydrogen). The first column (with nonpolar polymer sorbent Porapak Q) was used for separation of air (no separation for $\mathrm{O}_{2}$ and $\mathrm{N}_{2}$ ) and carbon dioxide (signals of positive polarity); the second column was used for separation of $\mathrm{O}_{2}, \mathrm{~N}_{2}$ and carbon monoxide (signals of negative polarity). Both sets of signals were summarized on the same chromatogram.

Figure 5. Gas chromatograms of gas phase under quercetin solution during its oxidation by air oxygen in a closed system: (a) - under initial quercetin suspension in the water ( $\mathrm{pH}=6.56)$; (b) - under basic solution of quercetin ( $\mathrm{pH} \sim 11$ ) after 4 days of contacting with air; (c) - after acidification of basic solution up to $\mathrm{pH}$ $\sim 1$.

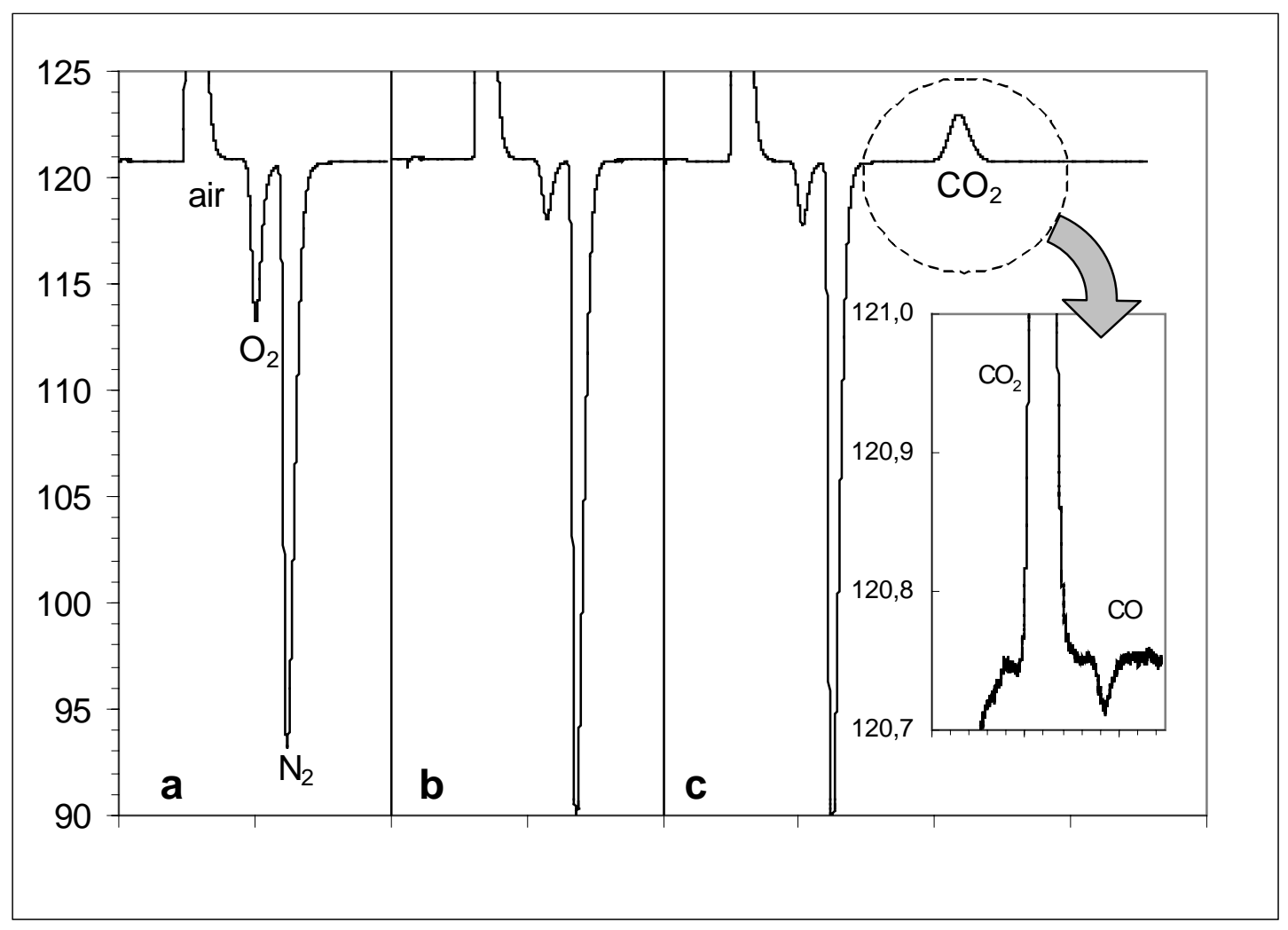

Figure 5a presents the gas phase chromatogram of a quercetin suspension in water at $\mathrm{pH}=6.56$, where the ratio of peak areas of $\mathrm{O}_{2}$ and $\mathrm{N}_{2}$ are equal to the content of these gases in the air (1:4). The 
chromatogram of the gas phase of an alkalinized solution of $\mathbf{1}$ in the water at $\mathrm{pH} \sim 11$ after 4 days of the contact with air in the closed system is shown in Figure 5b; the content of oxygen is visibly less, but no other gases $\left(\mathrm{CO}\right.$ or $\left.\mathrm{CO}_{2}\right)$ are detected. It is interesting that the total gas pressure in this system during the same time decreased from 1022 up to 930 mbar (- $9 \%$ ), which corresponds to the decrease of oxygen contents in the gas phase without formation of any volatile reaction products. Just this fact is enough to reject the previously proposed scheme for oxidative decarbonylation of quercetin [16]. Figure 5c presents the chromatogram of the gas phase after acidification of the solution of $\mathbf{1}$ with concentrated $\mathrm{HCl}$ up to $\mathrm{pH} \sim 1$; a carbon dioxide peak has appeared that is the direct confirmation of alternative mechanism of oxidative decarboxylation of quercetin (Scheme 5). However, the variations of the recorder scale allow us to reveal the presence of small amounts of carbon monoxide in the gas phase (negative signal with higher retention time than that of $\mathrm{CO}_{2}$ ). The peak areas of these two carbon oxides can be recalculated from the relative content of these reaction products in the gas phase with the use of coefficient $k=2.96$ (Table 5, see Experimental).

Table 5.

\begin{tabular}{|l|c|c|c|c|}
\hline $\begin{array}{c}\text { Gas phase } \\
\text { component }\end{array}$ & \multicolumn{3}{|c|}{ Peak areas, $\mathbf{S} \times \mathbf{1 0}^{-\mathbf{3}}, \mathbf{m V \times m s}$} & $\begin{array}{c}\text { Average } \\
\text { content in gas } \\
\text { phase, \% }\end{array}$ \\
\hline $\mathrm{CO}_{2}$ & 81.6 & 83.9 & 84.9 & $96.3 \pm 0.3$ \\
\hline $\mathrm{CO}$ & 1.18 & 1.04 & 1.06 & $3.7 \pm 0.3$ \\
\hline
\end{tabular}

Therefore, different mechanisms of quercetin transformation occur during its oxidation by air oxygen in moderately-basic water and water-ethanol solutions at ambient temperature. Under the mildest conditions of quercetin oxidation by air oxygen (at ambient temperature in moderately-basic media) the contribution of oxidative decarboxylation exceeds that of oxidative decarbonylation approximately by 26 times.

\section{Conclusions}

Oxidative destruction of quercetin by air oxygen under mild conditions should be considered as one of the typical reactions both for this compound and other flavonols. It is observed not only by heating its solutions in the presence of strong bases, but can take place in water and water-ethanol solutions in moderately-basic media $(\mathrm{pH} \sim 8-10)$ at ambient temperature without any radical initiators or irradiation of the reaction media. Revealed anomalies in the experimental results with dilute quercetin solutions in contact with air (e.g., stepwise titration) indicate that this process takes place even at $\mathrm{pH} \sim 7-8$, close to the $\mathrm{pH}$ values of physiological media. Therefore, the reaction under consideration may be one of the general biotransformation paths of quercetin and other flavonols, and this process may explain the low concentrations of these compounds in blood ( $\mathrm{pH} \sim 7.4$ ) in the presence of oxygen.

Direct head-space analysis of the gas phase of acidified quercetin solutions after air oxidation indicates that the principal gaseous reaction product under these conditions is carbon dioxide. Only small amounts of carbon monoxide (relative content ca. 4 \%) were detected. This means that the 
preferable mechanism is not oxidative decarbonylation of quercetin, but its oxidative decarboxylation. The possibility of facile oxidation of quercetin by air oxygen in solution should be taken into account in planning of all processes involving its isolation from plant raw materials and subsequent purification. Unusually large losses of quercetin during its re-crystallization from water solutions can be explained by this easy oxidation. Besides that, quercetin is widely used as a well known analytical reagent in determination of metal cations like $\mathrm{Fe}^{2+}, \mathrm{U}^{4+}$, in photometric determination of $\mathrm{Zr}$, Hf, Sc, Mo, Sn, Ge, in extraction-fluorescent analysis of $\mathrm{Hf}, \mathrm{Th}, \mathrm{Nb}, \mathrm{Ta}, \mathrm{Al}, \mathrm{Zr}$ and other elements. In all these cases the presence of quercetin oxidation products could distort the analytical results and, hence, should be excluded. The general practical recommendation is to avoid long-term contact of even weekly-prepared quercetin solutions with air.

\section{Experimental}

\section{General}

Commercially available quercetin dihydrate (CAS \# 6151-25-3) purchased from Sigma was used in all experiments. Determination of the solubility of (1) in the water-ethanol solutions (30 and $70 \%$ of ethanol) at ambient temperature $\left(21 \pm 1^{\circ} \mathrm{C}\right)$ was carried out by successive stepwise addition of 1 to known amounts of solvents $(60-70 \mathrm{~mL})$. The resulting values were $0.28 \mathrm{mg} / \mathrm{mL}$ (30 \% ethanol) and 3.5 $\mathrm{mg} / \mathrm{mL}$ (70 \% ethanol).

\section{Oxidation of $\mathbf{1}$ by air oxygen at ambient temperature}

Compound 1 (about $50 \mathrm{mg}$ ) was dissolved in $70 \%$ water-ethanol mixture (20 mL) placed in a 100 $\mathrm{mL}$ flask, followed by addition of aqueous ammonium hydroxide until the $\mathrm{pH}$ was $10.0 \pm 0.1$. Air was bubbled through this solution (with a flow rate of $c a .50 \mathrm{~mL} / \mathrm{min}$ ) using a mini-compressor for up to 3.5 hours. Aliquots of the reaction medium were taken periodically to record the UV-spectra (UVspectrometer Shimadzu UV-1700, wavelength range 200-500 nm) and for HPLC analyses. After termination of the process solvents were evaporated from the reaction mixtures at $100{ }^{\circ} \mathrm{C}$. The yields of dry products (deep red-brown powder) were ca. 50-70\%. For preparative syntheses the quantities of $\mathbf{1}$ and solvents were proportionally increased 3-5 fold. Oxidation of $\mathbf{1}$ at low temperatures $\left(1-2{ }^{\circ} \mathrm{C}\right)$ was conducted by cooling the reaction flask with ice. Additional purification of hydrophilic oxidation products from non-reacted residues of $\mathbf{1}$ included their dissolution in the minimal amount of water, filtering the less soluble quercetin and evaporation of the solvent.

\section{HPLC analyses of reaction mixtures and oxidation products}

HPLC analyses were carried out in two regimes: “quick" (1) and "smooth" (2). Regime (1) was performed using a Beckman System Gold LC chromatograph equipped with a UV-detector and a Luna C18 $150 \times 3.6 \mathrm{~mm}$ column (size of sorbent particles $5 \mu \mathrm{m}$ ) with a $20 \times 4.6 \mathrm{~mm}$ pre-column filled with the same sorbent. Elution regime: gradient (45 min) of 10 to $100 \%$ acetonitrile with a mixture of 0.03 $\%$ water solution of trifluoroacetic acid ( $\mathrm{pH} \approx 2.3$ ). Eluent flow $1.0 \mathrm{~mL} / \mathrm{min}$, injection size: $20 \mu \mathrm{L}$, detection wavelengths 254 and $360 \mathrm{~nm}$. Regime (2) involved the use of a Shimadzu LC-20 
chromatograph with a diode array detector and the same column/precolumn combination. Elution regime: gradient (60 $\mathrm{min}$ ) of 10 to $70 \% \mathrm{CH}_{3} \mathrm{CN}$ in the mixture with the same aqueous phase. Elution flow $1.0 \mathrm{~mL}$, injection size: $20 \mu \mathrm{L}$. UV-control of peak purity indicates the purity of all separated components.

\section{GC-MS analyses of oxidation products}

GC-MS analysis of the quercetin oxidation products was carried out with a HP-5890 gas chromatograph with a HP-5972 mass selective detector and a WCOT column (30 m $\times 0.25 \mathrm{~mm}$ with HP-5, film thickness $0.25 \mu \mathrm{m}$ ) under the following temperature programming regime: $30^{\circ} \mathrm{C}(5 \mathrm{~min}) \rightarrow$ ramp $7{ }^{\circ} \mathrm{C} / \mathrm{min} \rightarrow 300{ }^{\circ} \mathrm{C}$ (20 min). Injector temperature was $300{ }^{\circ} \mathrm{C}$, detector temperature $-280{ }^{\circ} \mathrm{C}$. Sample preparation included addition of methanol ( $c a .50 \mu \mathrm{L}$ ) to the dry sample $(2 \mathrm{mg})$, followed by the treatment of this mixture with bis-(trimethylsilyl)acetamide (BSA, $200 \mu \mathrm{L})$ at $80{ }^{\circ} \mathrm{C}(1 \mathrm{~min})$ until a homogenous solution was formed. Interpretation of GC-MS data was carried out with Wiley 138.L and NIST 98.L MS and GC retention indices databases. Mass spectra of principal components of reaction mixture; $\mathrm{m} / \mathrm{z}\left(\mathrm{I}_{\mathrm{rel}}>5 \%\right)$ and their retention times were as follows:

tris-TMS-phloroglucinol (A), $\mathrm{t}_{\mathrm{R}} 25.48 \mathrm{~min}: 344$ (10), 343 (25), 342 (81) M+, 341 (13), 328 (21), 327 (71), 268 (10), 253 (6), 147 (21), 133 (15), 99 (5), 75 (10), 74 (9), 73 (100), 45 (14).

tris-TMS-3,4-dihydroxybenzoic (protocatechuic) acid (B), $\mathrm{t}_{\mathrm{R}} 28.25 \mathrm{~min}: 371$ (7), 370 (21) M+, 355 (12), 311 (11), 281 (7), 267 (5), 223 (9), 195 (6), 194 (16), 193 (100), 165 (8), 137 (7), 74 (7), 73 (78), 45 (15).

tetrakis-TMS-2,4,6-trihydroxybenzoic acid (C), $\mathrm{t}_{\mathrm{R}} 30.72 \mathrm{~min}: 458$ (0.6) M+1, 446 (6), 445 (22), 444 (39), 443 (97), 369 (5), 355 (13), 283 (5), 147 (20), 133 (6), 75 (6), 74 (9), 73 (100), 45 (13).

\section{Gas chromatographic analysis of the gas phase of quercetin solutions during their oxidation}

The schematics of the device used for head-space analysis of the gas phase during quercetin oxidation is presented in Figure 6. It is closed system consisting of a glass vessel (3 L volume) filled with an aqueous quercetin suspension or solution ( $1 \mathrm{~L}$, volume of gas phase is therefore $2 \mathrm{~L}$ ) connected by steel tubes (i.d. $2 \mathrm{~mm}$ ) with the heated gas sampling valve (temperature $80^{\circ} \mathrm{C}$ ) for injecting of samples into the chromatograph and a digital pressure meter. The circulating pump provided continuous gas flow though the vessel containing the reaction media and the sampling valve with a flow rate of up to $0.8 \mathrm{~L} / \mathrm{min}$. Periodical injections of the gas phase were carried out manually, gas sample volume was $1 \mathrm{~mL}$, and the duration of the injection was $15 \mathrm{~s}$. Analyses were carried out on a Tsvett-500 (Tsvett Inc., Dzerzhinsk, Russia) gas chromatograph equipped with two packed columns placed in parallel and a thermoconductivity detector. Carrier gas was hydrogen with different flow rates through each column:

1. $2 \mathrm{~m} \times 2 \mathrm{~mm}$ with Porapak Q (Supelco, USA); carrier gas flow $17 \mathrm{~mL} / \mathrm{min}$;

2. $3 \mathrm{~m} \times 2 \mathrm{~mm}$ with $5 \AA$ molecular sieves (zeolites); carrier gas flow $42 \mathrm{~mL} / \mathrm{min}$.

Oven temperature was $40{ }^{\circ} \mathrm{C}$, detector temperature $150{ }^{\circ} \mathrm{C}$, filament current $100 \mathrm{~mA}$. Processing of GC signals was provided with MultiChrom software (version 1.5; Ampersand Co., Moscow, Russia). Both columns were connected to the single GC detector, so that components separated on column 1 
were registered on chromatogram as peaks of positive polarity, while components eluted from column 2 - appeared as peaks of opposite (negative) polarity. Average time of the analytical cycle did not exceed 15 min.

Figure 6. Scheme of the device used for head-space analysis of gas phase under quercetin solution during oxidation by air oxygen: 1 - vessel with solution; 2 - magnetic stirrer; 3 gas tubes; 4 - manometer connection; 5 - gas outlet connection; 6 - gas inlet connection; 7 - digital pressure meter; 8 - circulating pump; 9 - heated gas injection valve; 10 - gas sampling loop; 11 - carrier gas supply; 12 - gas chromatograph; 13 - packed column with molecular sieves (zeolites); 14 - packed column with Porapak Q.

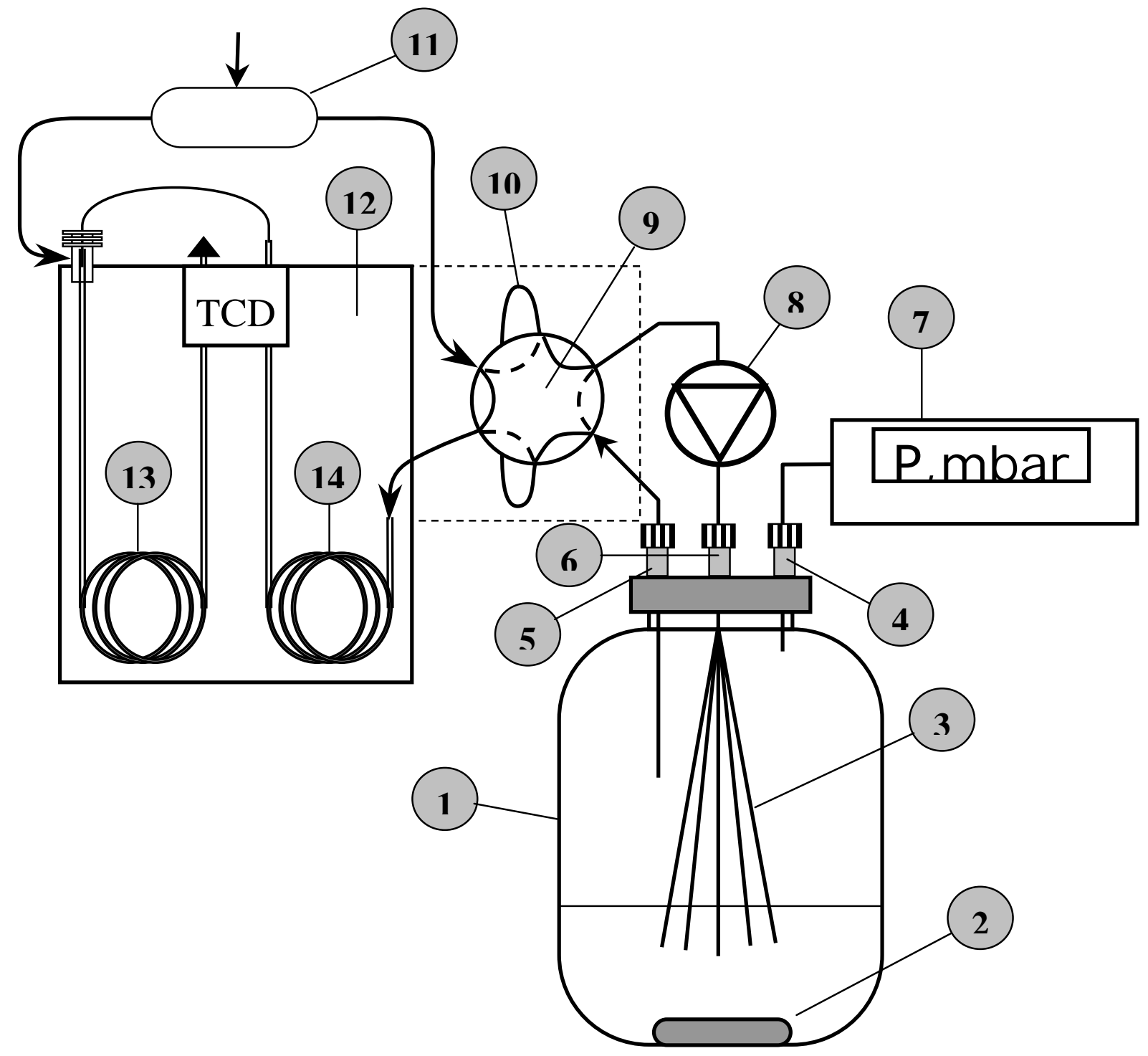

The ratio of air peak area (column 1) and the sum peak areas of $\mathrm{O}_{2}$ and $\mathrm{N}_{2}$ (column 2) gives the coefficient of relative sensitivity of the two channels of the detector $(k=2.96 \pm 0.01)$. To prevent the concentration of water vapor from gas phase on the sorbents, both columns should be periodically heated up to $130-150{ }^{\circ} \mathrm{C}$ (at least once per working day). 


\section{References}

1. Mabry, T.J.; Markham, K.R.; Thomas, M.B. The Systematic Identification of Flavonoids; Springer Verlag: N.Y. and Heidelberg, 1970.

2. Klysheva, L.K.; Bandukova, V.A.; Alunina, L.S. Plants Flavonoids. Spreading, Physicochemical Properties, Methods of Analysis (in Russian). Nauka Publ. House: Alma-Ata, 1978.

3. Andersen, O.M.; Markham, K.R., Eds. Flavonoids. Chemistry, Biochemistry and Applications. CRR Taylor \& Francis: N.Y., 2005.

4. Bors, W.; Hellers, W.; Michel, C. Flavonoids as Antioxidants: Determination of Radical Scavenging Efficiences. Meth. Enzymol. 1990, 186, 343-374.

5. Hanasaki, Y.; Ogawa, S.; Fukui, S. The Correlation between Active Oxygen Scavenging and Antioxidative effects of Flavonoids. Free Rad. Biol. Med. 1992, 16, 605-608.

6. Hu, J.P.; Calomme, M.; LAsure, A.; De Bryune, T.; Pieters, L.; Vlietnick A.; Van den Berghe, D.D.A. Structure-Activity Relationship of Flavonoids with Superoxide Scavenging Activity. Biol. Trace Elem. Res. 1995, 47, 327-331.

7. Rice-Evans, C.A.; Miller, N.J.; Pananga, G. Structure-Antioxidant Activity Relationships of Flavonoids and Phenolic Acids. Free Rad. Biol. Med. 1996, 20, 933-956.

8. Gulsen, A.; Makris, D.P.; Kefalas, P. Biomimetic Oxidation of Quercetin: Isolation of a Naturally Occuring Quercetin Heterodimer and Evaluation of its in vitro Antioxidant Properties. Food Res. Int. 2007, 40, 7-14.

9. Zenkevich, I.G.; Kosman, V.M. Relative Absorption at Different Wavelengths - Additional UVSpectral Parameter in Identification of Organic Compounds in Reversed-Phase HPLC (in Russian). Russ. J. Anal. Chem. 1996, 51, 870-875.

10. Kosman, V.M.; Zenkevich, I.G. Informational Maintenance of the Identification of Phenolic Compounds of Plant Origin in Reversed-Phase HPLC. Flavones, Flavonols. Flavonones and their Glicosides (in Russian). Plant Resources 1997, 33, 14-26.

11. Zenkevich, I.G.; Kochetova, M.V.; Larionov, O.G.; Revina, A.A.; Kosman, V.M. Retention Indices as the Most Reproducible Retention Parameters in Reversed Phase HPLC. Calculation for Hydrophylic Phenolic Compounds Using Reference n-Alkyl Phenyl Ketones. J. Liq. Chromatogr. Rel. Tech. 2005, 28, 2141-2162.

12. Zenkevich, I.G.; Pimenov, A.I. About Reasons of Anomalies in UV-Spectrum of Pentaacetylquercetin (in Russian). Plant Resources 2003, 39, 120-126.

13. Nierenstein, M.; Whedale, M. Anthocyanin. I. Anthocyanin-like Oxidation Products of Quercetin. Ber. Deut. Chem. Ges. 1912, 44, 3487-3491.

14. Matsuura, T.; Matsushima, H.; Sakamoto, H. Photosensitized Oxygenation of 3-Hydroxyflavones. Possible Model for Biological Oxygenation. J. Amer. Chem. Soc. 1967, 89, 6370-6371.

15. Matsuura, T.; Matsushima, H.; Nakashima, R. Photoinduced Reactions. XXXVI. Photosensitized Oxygenation of 3-Hydroxyflavones as a Nonenzymatic Model for Quercetinase. Tetrahedron 1970, 26, 435-443.

16. Brown, S.B.; Rajananda, V.; Holroyd, J.A.; Evans, E.G.V. A Study of the Mechanism of Quercetin Oxygenation by ${ }^{18}$ O Labelling. Biochem. J. 1982, 205, 239-244.

17. Kubo, I.; Nihey, K.; Shimizu, K. Oxidation Products of Quercetin Catalyzed by Mushroom Tyrosinase. Bioorg. Med. Chem. 2004, 12, 5343-5347. 
18. Ohashi, H.; Kyogoku, T.; Ishikawa, T.; Kawase, S.I.; Kawai, S. Antioxidative Activity of Tree Phenolic Constituents. I: Radical-Capturing Reaction of Flavon-3-ols with Radical Initiator. $J$. Wood Sci. 1999, 45, 53-63.

19. Krishnamachari, V.; Levine, L.H.; Pare, P.W. Flavonoid Oxidation by the Radical Generator AIBN: A Unified Mechanism for Quercetin Radical Scavenging. J. Agr. Food Chem. 2002, 50, 4357-4363.

20. Krishnamachari, V.; Levine, L.H.; Zhou, C.; Pare, P.W. In vitro Flavon-3-ol Oxidation Mediated by a B-Ring Hydroxylation Pattern. Chem. Res. Toxicol. 2004, 17, 795-804.

21. Makris, D.P.; Rossiter, J.T. Hydroxyl-Free Radical-Mediated Oxidative Degradation of Quercetin and Morin: A Preliminary Investigation. J. Food Composit. Anal. 2002, 15, 103-113.

22. Balogh-Hergovich, E.; Kaiser, J.; Speier, G. Synthesis and characterization of Copper (I) and Copper (II) Flavonolate Complexes with Phthalazine and Their Oxygenation and Relevance to Quercetinase. Inorg. Chim. Acta. 1997, 256, 9-14.

23. Balogh-Hergovich, E.; Kaiser, J.; Speier, G.; Argay, G.; Parkanyi, L. Kinetic Studies on the Copper (II)-mediated Oxygenolysis of the Flavonolate Ligand. Crystal Structure of [Cu(fla) $)_{2}$ (fla $=$ flavonolate) and $\left[\mathrm{Cu}(\mathrm{O}-\mathrm{bs})_{2}(\mathrm{py})_{3}\right](\mathrm{O}-\mathrm{bs}=\mathrm{O}-$ benzoylsalicylate). J. Chem. Soc. Dalton. Trans. 1999, 3847-3854.

24. Marfak, A.; Trouillas, P.; Allais, D.P.; Champavier, Y.; Calliste, C.A.; Duroux, J.L. Radiolysis of Quercetin in Methanol Solution: Observation of Depside Formation. J. Agr. Food Chem. 2002, 50, 4827-4833.

25. Marfak, A.; Trouillas, P.; Allais, D.P.; Calliste, C.A.; Duroux, J.L. Redox Reactions Obtained by $\gamma$-Irradiation of quercetin Methanol Solution are Similar to in vivo Metabolism. Radiat. Res. 2003, 159, 218-227.

26. Buchner, N.; Krumbein, A.; Rohn, S.; Kroh, L.W. Effect of Thermal Processing of the Flavonols Rutin and Quercetin. Rapid Commun. Mass Spectrom. 2006, 20, 3229-3235.

27. Makris, D.P.; Rossiter, J.T. Quercetin and Ritin (Quercetin 3-O-Rhamnosylglucoside) Thermal Degradation in Aqueous Media under Alkaline Conditions. Special Publ. - Roy. Soc. Chem. 2000, 248, 216-238.

28. Jorgensen L.V., Cernett C., Justesen U., Skibsted L.H., Dragsted L.O. Two-Electron Electrochemical Oxidation of Quercetin and Kaempferol Changes only the Flavonoid C-Ring. Free Rad. Res. 1998, 29, 339-350.

29. Zenkevich, I.G.; Eshchenko, A.Yu.; Makarova, S.V.; Makarov, V.G. Unusual Chemical Transformations of Natural Flavonoids // Abstr. 54 ${ }^{\text {th }}$ Annual Congr. on Medicinal Plant Research. 29 Aug.-2 Sept. 1006. Helsinki, Finland. № P300. Planta Med. 2006, 72, 1070.

30. Kuntic, V.; Pejic, N.; Micic, S.; Malesev, D.; Vujic, Z. Determination of Dissociation Constants of Quercetin. Pharmazie. 2003, 58, 439-440.

31. http://www.thermidaire.on.ca/do.html; http://cee.citadel.edu; http://www.usage.army.mil/ publications/eng-manuals/em1110-3-172/a-b.pdf.

32. Vesilind, P.A. Introduction to Environment Engineering; PWS Publishing Company: Boston, 1996.

33. Trouillas P., Marsal P., Siri D., Lazzaroni R., Duroux J.-L. A DFT Study of the Reactivity of OH Groups in Quercetin and Taxifolin Antioxidants. The Specificity of the 3-OH Site. Food Chem. 2006, 97, 679-688. 
34. Kutnevich, A.N.; Rudenko, A.P.; Rodina, L.L.; Pragst, F. Anion-Radicals from Cyclic Diketones with Alkyl Substituents (in Russian). Russ. J. Org. Chem. 1978, 14, 1343.

35. Korobitsina, I.K.; Gurevich, L.S.; Rodina, L.L. Chemistry of Ketones of Furanidine Series. IX. Alkaline Cleavage of 2,2,5,5-Tetraalkyl-3,4-furanidindiones (in Russian). Russ. J. Org. Chem. 1969, 5, 567-570.

36. Shimizu M., Orita H., Suzuki K., Hayakawa T., Hamakawa S., Takehira K. Oxidative C-C Bond Cleavage of vic-Diols with $\mathrm{H}_{2} \mathrm{O}_{2}$ Catalyzed by Heteropolyacids. J. Mol. Catal. A: Chem. 1996, 114, 217-220.

37. Frimer, A.A.; Gilinsky-Sharon, P. Reaction of Superoxide with Ascorbic Acid Derivatives: Insight into the Superoxide-Mediated Oxidation of Dehydroascorbic Acid. J. Org. Chem. 1995, 60, 2796-2801.

38. Ioffe, B.V.; Vitenberg, A.G. Head-Space Analysis \& Related Methods in Gas Chromatography; Wiley-Interscience Publishers: N.Y., 1984.

Sample Availability: Not applicable.

(C) 2007 by MDPI (http://www.mdpi.org). Reproduction is permitted for noncommercial purposes. 